\title{
Sarcome à cellules claires du rein : À propos d'un cas chez un jeune de 17 ans
}

\author{
Adil Mazdar, MD; Adil Ait Sakel, MD; Younes Essatara, MD; Ali Beddouche, MD; Hachem Elsayegh, MD; \\ Ali Iken, MD; Lounis Benslimane, MD; Yassine Nouini, MD
}

Service d'urologie «A,» CHU Rabat, Maroc

Cite as: Can Urol Assoc J 2014;8(5-6):e387-90. http://dx.doi.org/10.5489/cuaj.1748 Published online May 21, 2014.

\section{Résumé}

Le sarcome à cellules claires du rein (SCCR) se voit très rarement chez les jeunes. Il est caractérisé par une évolution agressive marquée par un taux élevé de récidive et de mortalité. Nous rapportons le cas d'un SCCR chez un patient de 17 ans et nous discutons de son apport et de son intérêt médical en vue d'une bonne prise en charge thérapeutique. L'agressivité du SCCR et la prolifération de métastases surtout osseuses impliquent qu'il ne faut pas méconnaître ce diagnostic afin de mettre en place un traitement adapté.

\section{Introduction}

Le sarcome à cellules claires du rein (SCCR) est une tumeur mésenchymateuse agressive, rare chez l'enfant (4\% des tumeurs rénales) et exceptionnelle chez l'adulte ${ }^{1}$. C'est l'une des tumeurs rénales agressives, dites à histologie défavorable, les plus fréquentes qui appartiennent au groupe des tumeurs du rein autres que la tumeur de Wilms ${ }^{1,2}$. Le SCCR doit être distingué du néphroblastome, qui est plus fréquent à cet âge et dont le traitement est différent ${ }^{3}$.

\section{Observation}

Nous rapportons le cas d'un patient âgé de 17 ans, sans antécédents pathologiques particuliers, présentant depuis 6 mois des douleurs au flanc droit sans autres signes associés.

Trois semaines avant son hospitalisation, le patient s'est plaint d'une intensification de la douleur avec augmentation progressive du volume de l'abdomen. L'examen clinique a permis de déceler la présence d'une masse tendue et sensible occupant l'épigastre, le flanc et I'hypocondre droit et s'étendant jusqu'à I'ombilic (fig. 1) avec $39,5{ }^{\circ} \mathrm{C}$ de fièvre.

L'examen biologique réalisé à I'admission a objectivé un syndrome infectieux biologique avec une CRP à 170 mg/l et une hyperleucocytose à $11200 \mathrm{~GB} / \mathrm{mm}^{3}$. Le taux d'hémoglobine était de $6 \mathrm{~g} / \mathrm{dl}$. Les autres valeurs étaient normales à I'examen biologique.

L'échographie abdominale a indiqué la présence $d$ 'une énorme masse tissulaire mesurant $25 \mathrm{~cm}$ de grand axe aux dépens du rein droit. La TDM abdomino-pelvienne a objectivé une masse tumorale polaire inférieure du rein droit, à développement antérieur majoritairement nécrosé. Cette masse présentait des contours bien délimités et était lobulée par endroits avec des plages hyperdenses suggérant des hémorragies et un envahissement de la graisse périrénale. II n'y avait pas d'envahissement des axes vasculaires ni des organes avoisinants (fig. 2, fig. 3, fig. 4).

Une antibiothérapie à large spectre par la ceftriaxone et la gentamicine a été entreprise, de pair avec une transfusion de culots globulaires. Devant l'instabilité hémodynamique, on a procédé à une néphrectomie totale élargie à visée hémostatique, mais aussi thérapeutique (fig. 5, fig. 6).

La TDM thoracique et la scintigraphie osseuse réalisées pendant la période postopératoire n'ont mis en évidence aucune anomalie.

L'étude histologique de la pièce opératoire a révélé la présence $d$ 'une prolifération cellulaire faite d'une nappe de cellules allongées aux noyaux atypiques se développant au sein d'un stroma lâche myxoïde (fig. 7, fig. 8). Cette prolifération a infiltré la capsule rénale, la graisse périrénale et le sinus. Les vaisseaux du hile et la recoupe urétérale étaient sains. Selon l'étude immunohistochimique, la tumeur exprimait la vimentine et le Bcl-2, cet aspect histologique correspondant à un sarcome à cellules claires.

\section{Discussion}

Le SCCR représente environ $4 \%$ des tumeurs rénales chez I'enfant et survient habituellement chez l'enfant âgé de 18 mois à 5 ans (30 mois en moyenne). Trait caractéristique, la fréquence des métastases osseuses a conduit les spécialistes à désigner en 1978 cette tumeur du groupe des néphroblastomes par le terme de « tumeur rénale de l'enfant métastasée 


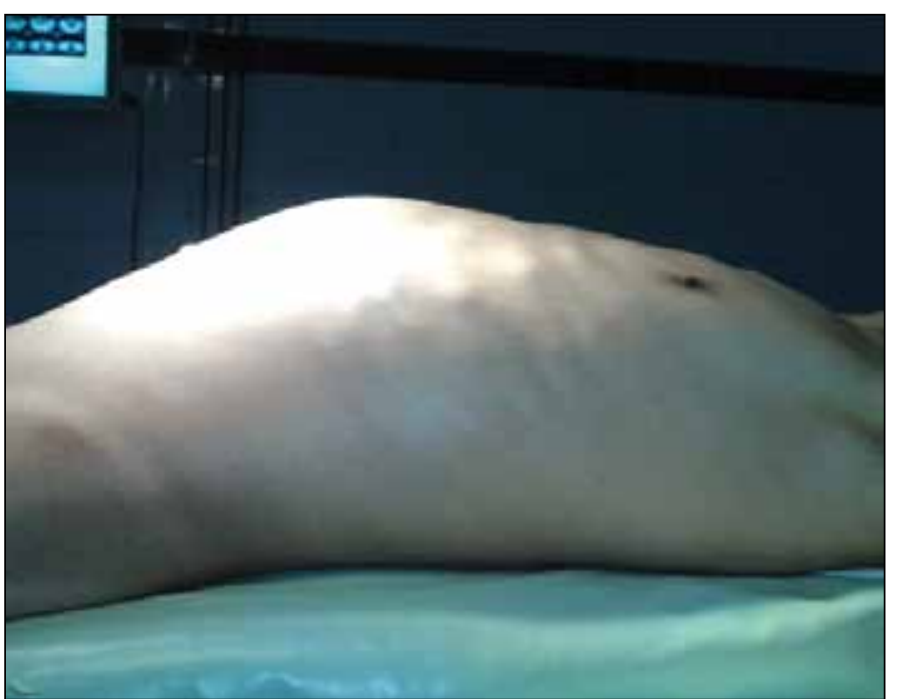

Fig. 1. Importante voussure de l'hypocondre droit.

aux $05 »^{4-6}$. Son histogénèse demeure à ce jour inconnue. Le pic d'incidence se situe entre 1 et 4 ans, avec des extrêmes allant de 3 jours à 57 ans. Le SCCR est exceptionnel chez l'adulte, avec une nette prédominance masculine?

Son mode de révélation clinique comporte habituellement la triade douleurs lombaires, hématurie et syndrome de masse, mais aussi une hypertension artérielle (HTA) ou de la fièvre (ou les deux $)^{8}$.

Les différents procédés d'imagerie permettent de mettre en évidence le processus tumoral, de réaliser une cartographie préopératoire et d'effectuer un bilan d'extension ${ }^{7,8}$. Ces tumeurs n'ont pas de caractère spécifique et ne peuvent pas être différenciées d'autres cancers du rein, voire de tumeurs primitives du rétropéritoine de type sarcome lorsqu'elles ont un développement exorénal prédominant ${ }^{9}$. L'échographie confirme l'origine rénale de la tumeur, qui

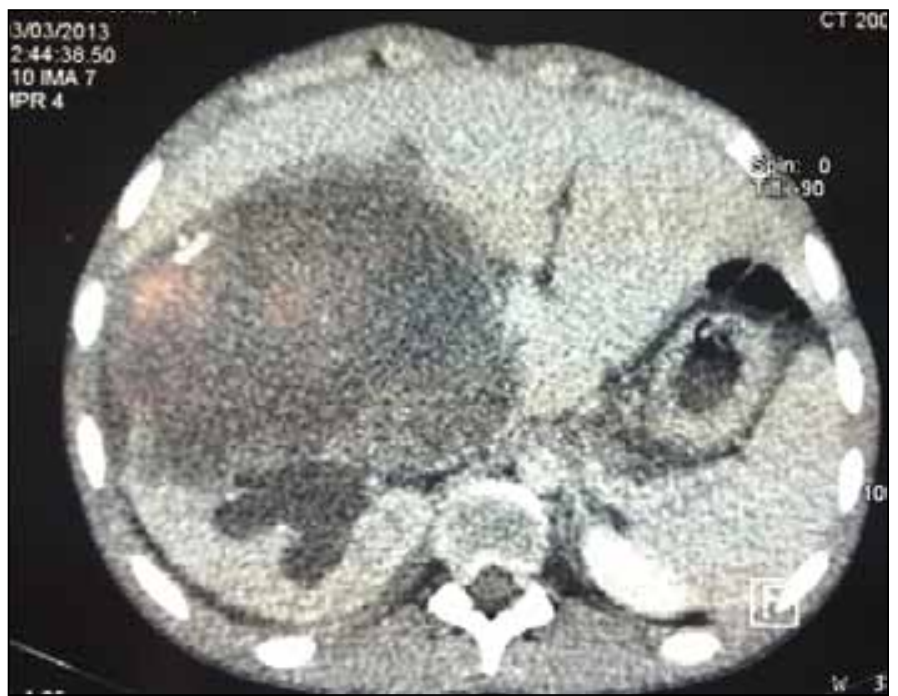

Fig. 3. Aspect tomodensitométrique en temps artériel d'une masse abdominale volumineuse et hétérogène aux dépens du rein droit.

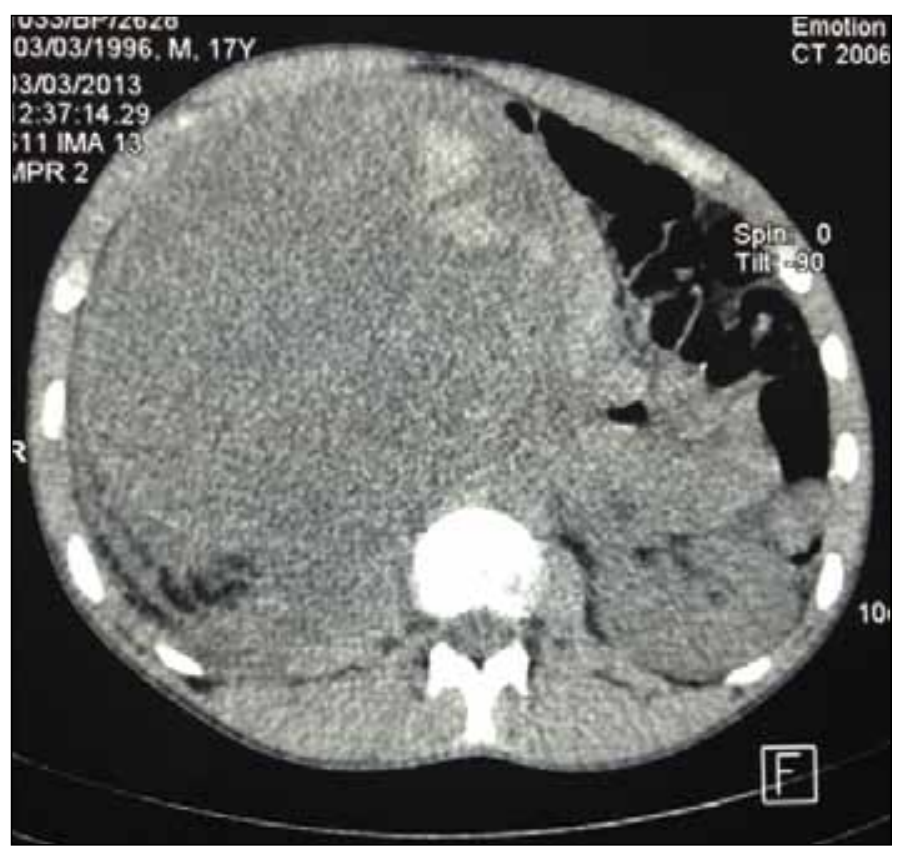

Fig. 2. Aspect tomodensitométrique sans injection d'une volumineuse masse abdominale, non encapsulée, de densité hétérogène avec des zones hyperdenses indiquant la présence de foyers hémorragiques et dont les rapports avec le rein sont imprécis.

se présente habituellement sous la forme d'une masse volumineuse comportant des zones nécrotiques ou surtout kystiques. Le doppler permet d'évaluer la perméabilité de la veine cave inférieure et l'extension à la veine rénale, celle-ci pouvant être le siège $d^{\prime}$ un thrombus dans $5 \%$ des $\mathrm{SCCR}^{10}$. La TDM confirme les données de l'échographie et permet de mieux étudier les rapports vasculaires et locorégionaux. Elle révèle la présence d'une masse hétérogène dont la densité est légèrement inférieure à celle du parenchyme avoisinant et pouvant comporter des zones hémorragiques ${ }^{11}$. L'injection

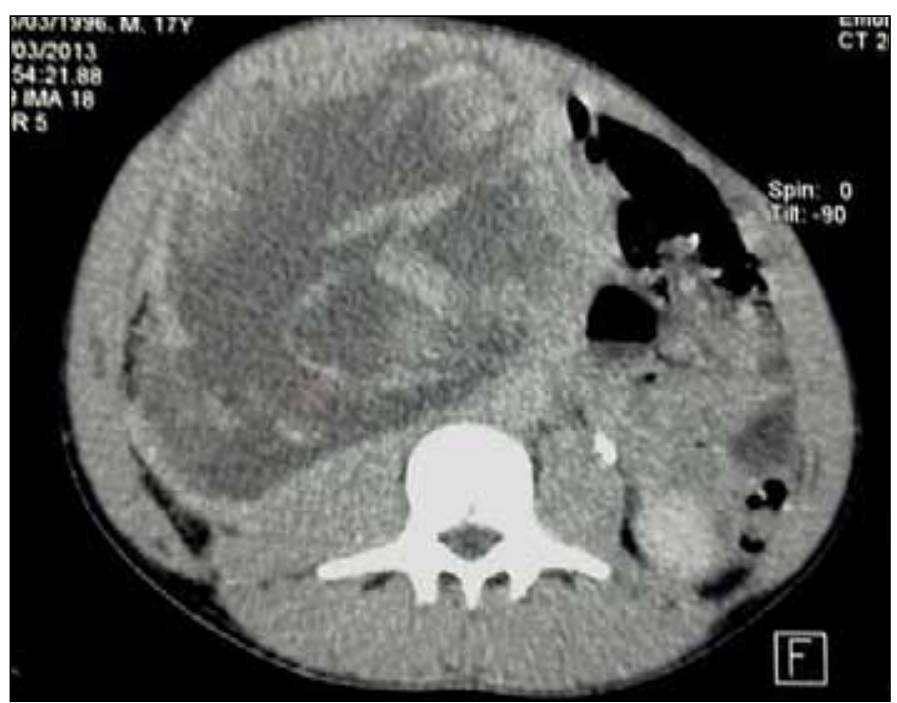

Fig. 4. Aspect tomodensitométrique en temps tardif montrant une masse du rein droit qui prend le contraste de façon hétérogène. 


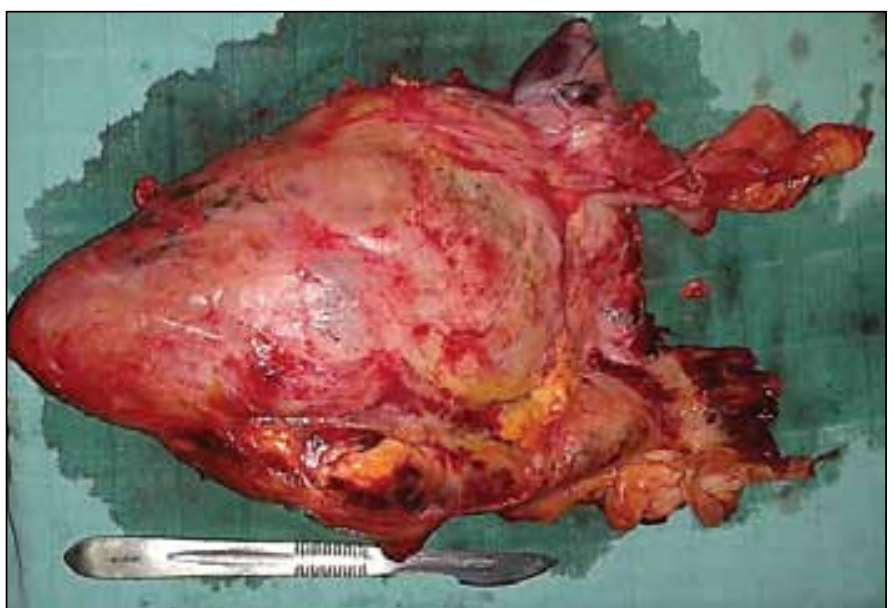

Fig. 5. Aspect macroscopique en vue postérieure de la tumeur sur pièce de néphrectomie : masse d'aspect myxoïde et blanchâtre de très grande taille.

intraveineuse de produit de contraste permet d'apprécier le degré de vascularisation de la masse tumorale, qui est très variable. La TDM objective également, comme pour toute tumeur maligne du rein, un envahissement de la veine rénale ou de la veine cave inférieure par la découverte d'une hypodensité intravasculaire ${ }^{9}$. L'IRM, si elle est pratiquée, permet d'analyser avec beaucoup de précision les rapports vasculaires de la masse. Un bilan d'extension issu particulièrement $d^{\prime}$ une scintigraphie osseuse est recommandé, $d u$ moment que les métastases osseuses sont présentes dans de $40 \%$ à $70 \%$ des $\operatorname{cas}^{10}$.

Sur le plan anatomopathologique, le SCCR se présente macroscopiquement sous forme surtout $d^{\prime}$ une tumeur unilatérale médiorénale de grande taille (taille moyenne de 11 $\mathrm{cm})$, bien délimitée, mais non encapsulée, ne dépassant généralement pas la capsule rénale. La limite tumeur-parenchyme rénal est bien définie. La tumeur présente un aspect brunâtre et une consistance molle. Un aspect mucoïde

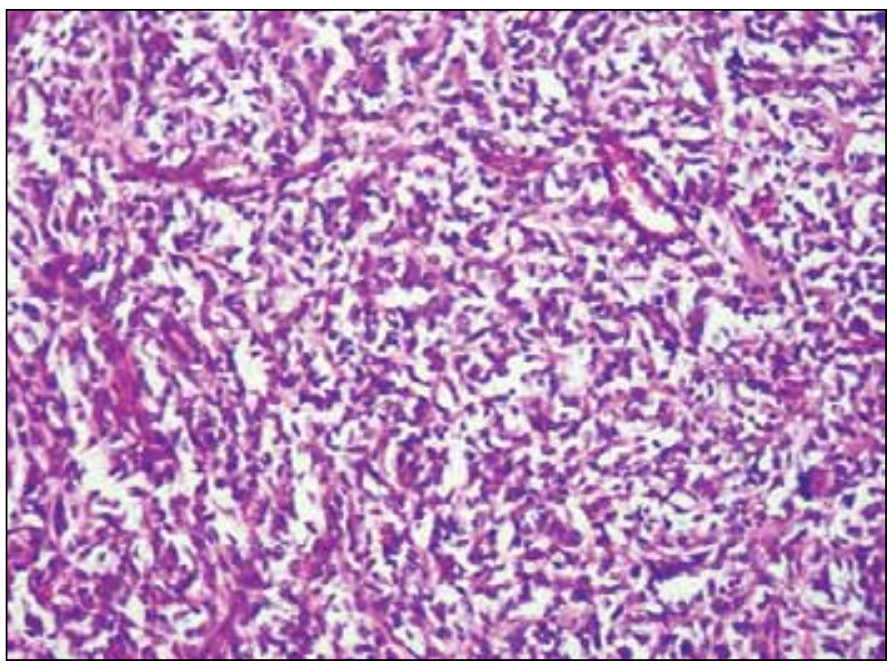

Fig. 7. Stroma fibrovasculaire grêle donnant un aspect alvéolaire ou trabéculaire à la prolifération tumorale $(\mathrm{HE} \times 20)$.

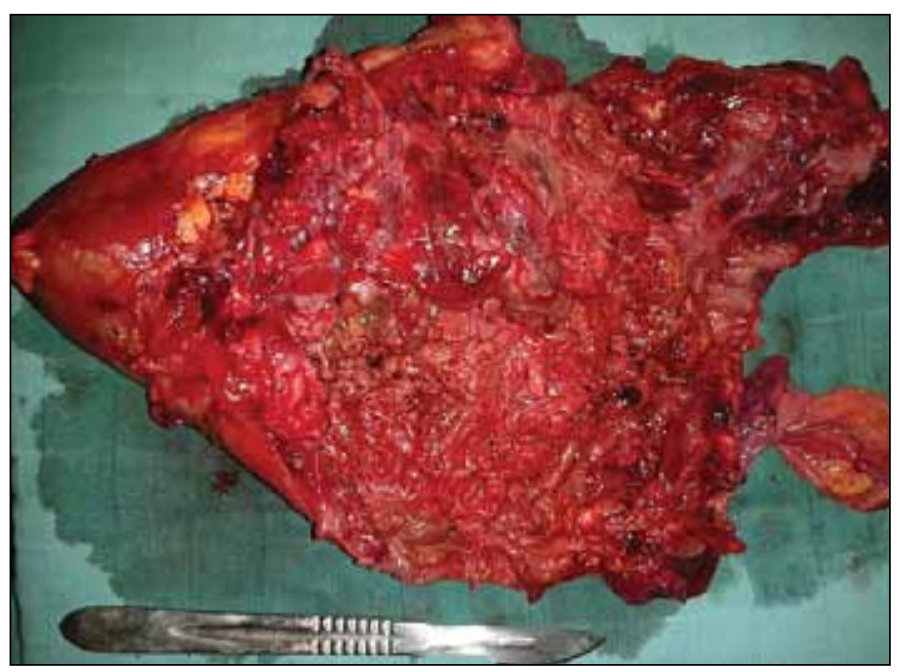

Fig. 6. Aspect macroscopique en vue antérieure de la tumeur sur pièce de néphrectomie : masse très hétérogène avec rupture capsulaire et infiltration du rein.

est parfois observé, de même que des remaniements kystiques ${ }^{4,12}$. Les kystes peuvent parfois être prédominants, ce qui permet de suspecter radiologiquement un néphrome kystique ou un kyste rénal multiloculaire ${ }^{12}$.

Histologiquement, la forme classique du SCCR se caractérise par une prolifération tumorale agencée en nids ou en cordons typiquement séparés par un réseau vasculaire fait de capillaires branchés comme celui du liposarcome. Les cellules tumorales, fusiformes ou ovoïdes, à cytoplasme clair, aux limites mal visibles, sont munies de noyaux ronds ou ovalaires, pourvus d'une chromatine fine, sans nucléole apparent avec un aspect vitreux, optiquement vide, ce qui est un excellent signe pour le diagnostic, tout comme l'abondante matrice extracellulaire, mucopolysaccharidique, qui contribue elle aussi à l'aspect pâle de ces tumeurs et qui justifie l'emploi du terme "sarcome à cellules claires $»^{13}$. En

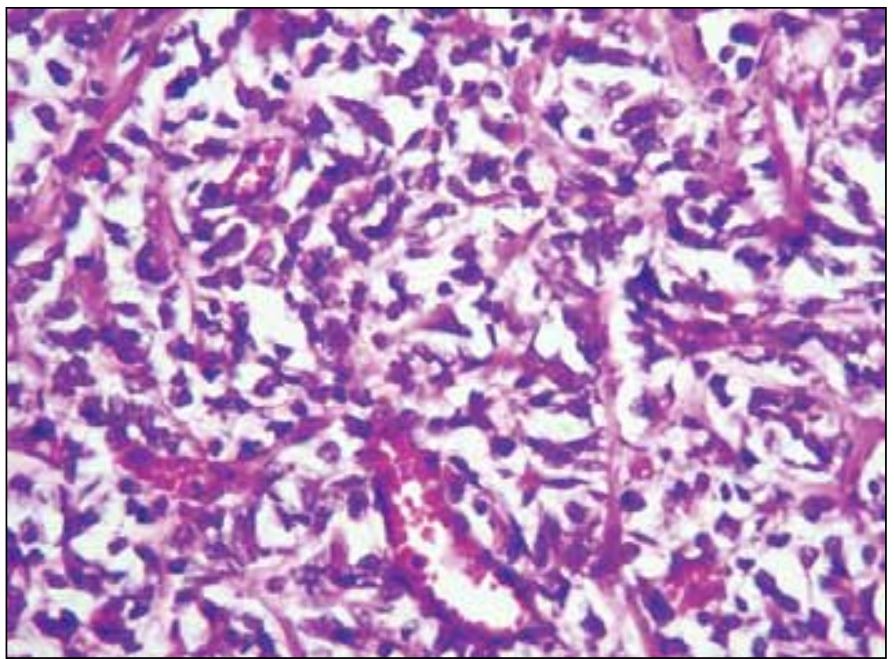

Fig. 8. Les cellules tumorales sont pourvues d'un noyau rond ou ovalaire et de cytoplasmes peu abondants. Les mitoses sont peu fréquentes (HE $\times 40$ ). 
dehors de la forme classique, qui est visible au moins focalement dans plus de $90 \%$ des cas, il existe plusieurs variantes morphologiques qui peuvent être plus ou moins associées dans une même tumeur : myxoïde (50\% des SCCR), sclérosante (35\% des SCCR), cellulaire (25\% des SCCR), épithélioïde (13\% des SCCR), palissadique (15\% des SCCR), fusiforme ( $7 \%$ des SCCR) et sinusoïdale ${ }^{4}$. Toutefois, ces variantes histologiques $\mathrm{n}^{\prime}$ ont pas de valeur pronostique.

En immunohistochimie, seuls la vimentine et le bcl-2 sont positifs. Les marqueurs épithéliaux, y compris l'EMA, ne sont pas exprimés même dans la variante épithéliö̈de. Ils ne sont exprimés qu'au niveau des tubes rénaux résiduels enchâssés dans la prolifération tumorale. L'antigène CD34, la PS100 et la desmine sont négatifs ${ }^{4,8,11}$.

Le traitement des sarcomes à cellules claires du rein chez I'adulte est l'objet de controverse : chirurgie, radiothérapie ou chimiothérapie, utilisées seules ou en association. En effet, le sarcome à cellules claires du rein est rare, rapporté sous forme de cas sporadiques et ayant des protocoles thérapeutiques différents, ce qui rend la prise en charge thérapeutique optimale difficile à préciser ${ }^{14}$. À ce jour, peu d'études ont porté sur la prise en charge thérapeutique. Le traitement actuel, selon le protocole SIOP 2001, préconise une néphrectomie élargie, la radiothérapie et une polychimiothérapie intensive et prolongée à base d'agents alkylants, d'anthracyclines, d'épipodophyllotoxines et de dérivés du platine $^{8}$.

L'évolution reste marquée par la fréquence de métastases ganglionnaires périrénales au moment du diagnostic $(29 \%)^{4,6}$. Les métastases osseuses ostéolytiques représentent le mode de récidive le plus fréquent, variant de $18,6 \%$ à $70 \%$ des cas et survenant même après 10 ans d'évolution. Les sites de prédilection sont essentiellement le crâne, le fémur, les côtes et les vertèbres ${ }^{4,6}$. Le SCCR a tendance donc à infiltrer le rein et l'aire lymphovasculaire périrénale, ce qui explique la fréquence des récidives locorégionales et la nécessité d'une surveillance tomodensitométrique tous les trois mois pendant les deux premières années, puis deux fois par $a^{7}$. Le taux de survie global associé au SCCR est de l'ordre de $69 \%$, avec un taux de survie sans récidive de $60 \%{ }^{6}$.

Le pronostic du SCCR reste sombre. Des facteurs pronostiques ont été reconnus comme significatifs et indépendants dans une large série de 351 patients : le stade avancé, l'âge et la présence de nécrose seraient des éléments de mauvais pronostic, alors qu'un traitement à base de doxorubicine serait un élément améliorant la survie ${ }^{6}$.

\section{Conclusion}

Le SCCR est une tumeur maligne très rare chez I'adulte, de pronostic sombre. Son diagnostic relève de l'examen histologique. L'imagerie moderne est d'un intérêt capital, car elle permet d'effectuer des biopsies guidées et un bilan d'extension précis. Le traitement est encore à ce jour l'objet de controverse : chirurgie, radiothérapie ou chimiothérapie, utilisées seules, mais souvent en association. L'agressivité du SCCR et la prolifération des métastases surtout osseuses qui l'accompagne impliquent qu'il ne faut pas méconnaître ce diagnostic afin de mettre en place un traitement adapté. Nous insistons sur la nécessité de mettre au point des médicaments cytotoxiques plus efficaces afin d'améliorer le pronostic.

Competing interests: Dr. Mazdar, Dr. Sakel, Dr. Essatara, Dr. Beddouche, Dr. Elsayegh, Dr. Iken, Dr. Benslimane and Dr. Nouini all declare no competing financial or personal interests.

This paper has been peer-reviewed.

\section{Références}

1. Broecker B. Non-Wilms' renal tumors in children. Urol Clin North Am 2000;27:463-9. http://dx.doi. org/10.1016/S0094-0143(05)70094-X

2. Kidd JM. Exclusion of certain renal neoplasms from the category of Wilms' tumor. Am J Pathol 1970;59:16a

3. Huang $C C$, Cutcliffe $C$, Coffin $C$, et al. Classification of malignant pediatric renal tumors by gene expression. Pediatr Blood Cancer 2006;46:728-38. http://dx.doi.org/10.1002/pbc.20773

4. Eble JN, Sauter G, Epstein II, et al. World Health Organisation Classification. Pathology and genetics. tumours of the urinary system and male genital organs. Lyon: IARC Press; 2004.

5. Marsden HB, Lawler W, Kumar PM. Bone metastasizing renal tumor of childhood: morphological and clinical features, and differences from Wilms' tumor. Cancer 1978;42:1922-8. http://dx.doi. org/10.1002/1097-0142(197810)42:4<1922::AID-CNCR2820420434>3.0.C0;2-Z

6. Argani P, Perlan EJ, Breslow NE, et al. A review of 351 cases from the national Wilms tumor study group pathology center. Am I Surg Pathol 2000;24:4-18. http://dx.doi.org/10.1097/00000478200001000-00002

7. Benchekroun $A$, Ghadouane $M$, Zannoud $M$, et al. Clear cell sarcoma of the kidney in an adult. A case report. Ann Urol (Paris) 2002;36:33-5. http://dx.doi.org/10.1016/S0003-4401 (01)00080-8

8. Namuouia RY, Castexb MP, Vialc J, et al. Sarcome à cellules claires du rein : à propos d'un cas Pédiatrique. Prog Urol 2010;20:465-8. http://dx.doi.org/10.1016/i.purol.2009.09.038

9. Adnani A, Latib R, Bouklata $S$, et al. Sarcome à cellules claires du rein chez I'adulte : à propos d'un cas. J Radiol 2006;87:136-8. http://dx.doi.org/10.1016/S0221-0363(06)73985-3

10. Glass RB, Davidson AJ, Fernbach SK. Clear cell sarcoma of the kidney. Radiology 1991;180:715-7.

11. Shirkhoda A, Errol L. Renal sarcoma and sarcomatoid renal cell carcinoma: CT and angiographic features. Radiology 1987;162:353-7.

12. Murphy WM, Grignon DJ, Perlman EJ. AFIP atlas of tumor pathology (fourth series, fascicle 1). Tumors of the kidney bladder, and related urinary structures. Washington: American Registry of Pathology; 2004.

13. Sassi $\mathrm{SH}$, Braham E, Oubiche $\mathrm{F}$, et al. Sarcome à cellules claires du rein. À propos de deux cas pédiatriques. Ann Pathol 2008;28:36-40. http://dx.doi.org/10.1016/j.annpat.2008.03.002

14. Kitamura H, Fujimoto H, Tobisu K, et al. Dynamic computed tomography and color doppler ultrasound of renal parenchymal neoplasms: Correlations with histopathological findings. Jpn J Clin Oncol 2004;34:7881. http://dx.doi.org/10.1093/iico/hyh013 